\title{
An Adaptation of the Scheifele Method to Stiff Systems of Differential Equations
}

\author{
J.A. Reyes, F. García-Alonso and Y. Villacampa*
}

Department of Applied Mathematics. Higher Polytechnic School (EPS). University of Alicante, Spain

\begin{abstract}
Many branches of Physics and Engineering use perturbed linear ODE's. One method of resolution is based on the use of Scheifele functions for systems. This paper systematically expands three applications of the Scheifele method adapted to stiff problems. For this purpose, a family of matrices, $\Gamma$-functions and the numerical method are presented for the integration of perturbed linear systems with constant coefficients, which enables the solution to be expressed as a series of $\Gamma$-functions. The series coefficients are obtained through recurrence relations involving the perturbation function. One of the main difficulties in terms of implementing the method is the need to determine these relations for each case.

Furthermore, in this paper, the necessary adaptations are made in order to facilitate the calculation of the recurrence relations required for system integration. In each problem, the numerical algorithm is designed with a view to enabling computational implementation. This algorithm presents the same good properties as the integration method for harmonic oscillators, in other words it can accurately integrate the non-perturbed problem using just the first term in the series.

The results show increased accuracy in the application of the model when compared to other known methods implemented in Maple V.
\end{abstract}

Keywords: Numerical solutions for perturbed linear ODE systems; Stiff problems.

\section{INTRODUCTION}

In 1971, Scheifele [1] constructed a one-step numerical method for the integration of perturbed oscillators, a problem that frequently appears in many branches of Physics and Engineering. This method is based on the construction of a family of functions, $G_{k}$, which enables the solution to the oscillator to be expressed in terms of the series $x(t)=\sum_{k=0}^{\infty} b_{k} G_{k}[2]$ and also offers the advantage of being able to integrate accurately the non-perturbed oscillator using just the first two terms. The generalization of this method to perturbed first-order linear differential equation systems can be found in [3], where a new family of functions, $\Gamma_{k}$, is constructed, adapted to the integration of this type of system. The solution in terms of $\Gamma$-functions is given by the series $\boldsymbol{x}(t)=\sum_{k=0}^{\infty} \Gamma_{k} \mathbf{b}_{k}$, where the coefficients $\mathbf{b}_{k}$ are obtained using recurrences involving the perturbation function of the system. This means that the $\Gamma$-functions series method for systems presents difficulties in terms of its application particularly when the perturbation function has a complicated analytical expression. However, given its good performance in relation to stiff problems and highly oscillatory problems, the $\Gamma$-functions method is crucial for the construction of multistep algorithms that solve this drawback, such as the SMF [4-6], SVF [7] and $\mathrm{EI}_{\mathrm{p}} \mathrm{PC}$ [8] methods, among

*Address correspondence to this author at the Department of Applied Mathematics Higher Polytechnic School (EPS). University of Alicante, Spain; E-mail: villacampa@ua.es others. Furthermore, the $\Gamma$-functions series method retains the good properties displayed by the series method in relation to differential equations, in other words, if at a specific time $t$ the perturbation terms disappear, the $\Gamma$-functions series method will accurately integrate the homogenous problem using just the first term in the series.

In addition to provide a detailed presentation of $\Gamma$ functions and their most relevant properties as well as the calculation of residuals, this paper systematically expands three applications of the method to the integration of stiff systems. In these problems, all the necessary adaptations are made in order to facilitate their integration, applying Steffensen techniques $[9,10]$ and defining in each of them the numerical algorithm that allows for computational implementation. Finally, the relative error for the solutions obtained using the $\Gamma$-functions method is compared with that of solutions provided by other well-known codes such as LSODE, MGEAR and GEAR, implemented in Maple V.

\section{GENERATION AND PROPERTIES OF $\Gamma$ - FUNC- TIONS}

Let us consider the following initial value problem, IVP:

$x^{\prime}=A x+\varepsilon f(x, t), \quad x(0)=x_{0}$

where $A$ is a regular $n$-order matrix, $\varepsilon$ is a small perturbation parameter and the components of vector $\boldsymbol{x}$ are functions $x_{i}(t)$ with $i=1,2, \ldots, n$. The components of the vector perturbation field $\boldsymbol{f}(\boldsymbol{x}, t)$ are $f_{i}(\boldsymbol{x}, t)$ with $i=1,2, \ldots, n$ and the field is continuous, with continuous derivatives until a certain order so that it satisfies the conditions for existence 
and uniqueness of the solution. This type of system is called a perturbed linear system.

Let us suppose that the perturbation function $\boldsymbol{g}(t)=\boldsymbol{f}(\boldsymbol{x}, t)$ admits the following series expansion:

$\boldsymbol{f}(\boldsymbol{x}, t)=\sum_{k=0}^{\infty} \frac{t^{k}}{k !} \mathbf{c}_{k+1}$,

In this case, the initial value problem can be expressed as follows:

$\boldsymbol{x}^{\prime}=A \boldsymbol{x}+\varepsilon \sum_{k=0}^{\infty} \frac{t^{k}}{k !} \mathbf{c}_{k+1}, \quad \boldsymbol{x}(0)=\boldsymbol{x}_{0}$.

The solution to IVP (3) can be obtained by applying the superposition principle, in other words, by constructing the general solution for the homogeneous system using the initial condition given and adding a solution to the complete problem with a null initial condition.

The solution to the complete problem with a null initial condition can be obtained by resolving the following individual IVP's:

$\left(\underline{x}_{j+1}^{k}\right)^{\prime}=A \underline{x}_{j+1}^{k}+\frac{t^{j}}{j !} \underline{e}^{k}$,

$\underline{x}_{j}^{k}(0)=\underline{0} \quad j=0,1,2, \ldots \quad k=1,2, \ldots, n$,

where:

$\underline{x}_{j}^{k} \in M(n, 1 ; \mathbb{R})$,

$\underline{e}^{k}=(0, \ldots, 1, \ldots, 0)^{T}$,

and effectively combining them with coefficients $\mathcal{E}$ and $\mathbf{c}_{k}$.

In order to simplify the notation, squared $n$-order matrices, $X_{j}$, are introduced, in which the columns are matrices $\underline{x}_{j}^{k}$, in other words:

$X_{j}=\left(\underline{x}_{j}^{1}, \underline{x}_{j}^{2}, \ldots, \underline{x}_{j}^{n}\right)$ with $j=0,1,2, \ldots$. IVP:

Clearly, these matrices are solutions to the following

$X_{j}^{\prime}=A X_{j}+\frac{t^{j}}{j !} I, \quad X_{j}(0)=\underline{0} \quad, \quad j=0,1, \ldots$.

where $X_{j}$ is a real function with values on ring $\mathcal{M}(n, \mathbb{R})$ of the squared $n$-order matrices, in which $I$ and $\underline{0}$ are the unity and neutral elements of this ring, respectively.

The solutions to (7) are the Scheifele $\Gamma$-functions [3] and are expressed as:

$X_{j}(t)=\Gamma_{j+1}(t), j=0,1, \ldots$.

Although the notation does not indicate this explicitly, these functions depend on $A$.

The $\Gamma$-functions obey:
$\Gamma_{j}^{\prime}(t)=\Gamma_{j-1}(t) \quad j=2,3, \ldots$.

By using the differentiation rule (9), the first $\Gamma$-function is defined, $\Gamma_{0}(t)$, is given by:

$\Gamma_{0}(t)=\Gamma_{1}^{\prime}(t)$.

Taking into account (8), (9) and (10), the following recurrence relation is obtained:

$\Gamma_{j}(t)=A \Gamma_{j+1}(t)+\frac{t^{j}}{j !} I, \quad j=0,1, \ldots$

The importance of this recurrence relation lies in its application to the calculation of $\Gamma$-functions

From (10) and (11), it can be deduced that the function $\Gamma_{0}$ is the solution to the following IVP:

$x^{\prime}=A x, \quad x(0)=I$,

in other words:

$\Gamma_{0}^{\prime}(t)=A \Gamma_{0}(t)$ and $\Gamma_{0}(0)=I$.

Another significant property in relation to the calculation of $\Gamma$-functions is that such functions can be expressed using series expansions:

$\Gamma_{j}(t)=\sum_{k=0}^{\infty} \frac{t^{k+j}}{(k+j) !} M_{k}, \quad M_{k} \in \mathcal{M}(n, \mathbb{R})$.

By analytically expanding the function $\Gamma_{0}$, we see that:

$\Gamma_{0}(t)=\sum_{k=0}^{\infty} \frac{t^{k}}{k !} M_{k}$, where $M_{k} \in \mathcal{M}(n, \mathbb{R})$.

By taking the derivative of the above expression, we get:

$\Gamma_{0}^{\prime}(t)=\sum_{k=1}^{\infty} \frac{t^{k-1}}{(k-1) !} M_{k}=\sum_{k=0}^{\infty} \frac{t^{k}}{k !} M_{k+1}$,

by (12) and (13)

$\sum_{k=0}^{\infty} \frac{t^{k}}{k !} M_{k+1}=A \sum_{k=0}^{\infty} \frac{t^{k}}{k !} M_{k}=\sum_{k=0}^{\infty} \frac{t^{k}}{k !} A M_{k}$,

by identifying these terms, we get:

$M_{k+1}=A M_{k}, \quad k=0,1, \ldots$.

and hence:

$M_{k}=A^{k} M_{0}, \quad k=0,1, \ldots$.

On the basis of (12) and (13), and having evaluated $\Gamma_{0}(t)$ as $t=0$, we can deduce that:

$M_{0}=I$,

$M_{k}=A^{k}, \quad k=1, \ldots$,

then: 
$\Gamma_{0}(t)=\sum_{k=0}^{\infty} \frac{t^{k}}{k !} A^{k}=e^{t A}$,

A particular solution to the problem:

$\boldsymbol{x}^{\prime}=A \boldsymbol{x}+\varepsilon \sum_{k=0}^{\infty} \frac{t^{k}}{k !} \mathbf{c}_{k+1}, \quad \boldsymbol{x}(0)=\underline{0}$,

is:

$\boldsymbol{x}_{p}=\varepsilon \sum_{k=1}^{\infty} \Gamma_{k}(t) \mathbf{c}_{k}$.

The solution for the IVP:

$\boldsymbol{x}^{\prime}=A \boldsymbol{x}+\varepsilon \boldsymbol{f}(\boldsymbol{x}, t), \quad \boldsymbol{x}(0)=\boldsymbol{x}_{0}$,

takes on the form:

$\boldsymbol{x}(t)=\Gamma_{0}(t) \boldsymbol{x}_{0}+\varepsilon \sum_{k=1}^{\infty} \Gamma_{k}(t) \mathbf{c}_{k}$.

This result enables the numerical integration to be defined on the basis of $\Gamma$-functions.

\section{FINITE EXPANSIONS IN $\Gamma$-FUNCTIONS AND THE CALCULATION OF RESIDUALS}

By performing a Taylor series expansion and truncating the solution $\boldsymbol{x}(t)$ for IVP (1), an approximation of the solution can be obtained, as follows:

$\boldsymbol{x}_{m}(t)=\sum_{k=0}^{m} \frac{t^{k}}{k !} \mathbf{a}_{k}$ with $\mathbf{a}_{k}=\boldsymbol{x}^{k)}(0) \in \mathbb{R}^{n}$.

By replacing expression (11) in (26) and taking into account that matrices $\Gamma_{k}(t)$ and $A$ commute, the following can be expressed:

$\boldsymbol{x}_{m}(t)=\Gamma_{0}(t) \mathbf{a}_{0}$

$+\sum_{k=0}^{m-1} \Gamma_{k+1}(t)\left(\mathbf{a}_{k+1}-A \mathbf{a}_{k}\right)-\Gamma_{m+1}(t) A \mathbf{a}_{m}$.

By defining a new sequence of coefficients as:

$\mathbf{b}_{0}=\mathbf{a}_{0}$,

$\mathbf{b}_{k+1}=\mathbf{a}_{k+1}-A \mathbf{a}_{k}$,

(27) is reduced to:

$\boldsymbol{x}_{m}(t)=\sum_{k=0}^{m} \Gamma_{k}(t) \mathbf{b}_{k}-\Gamma_{m+1}(t) A \mathbf{a}_{m}$. proximation:

Eliminating the final term gives a different ap-

$\boldsymbol{X}_{m}(t)=\sum_{k=0}^{m} \Gamma_{k}(t) \mathbf{b}_{k}$,

which provides greater accuracy than

$\mathbf{x}_{m}(t)=\sum_{k=0}^{m} \frac{t^{k}}{k !} \mathbf{a}_{k}$.
In fact, the coefficients in the expression of $\boldsymbol{x}_{m}(t)=\sum_{k=0}^{m} \frac{t^{k}}{k !} \mathbf{a}_{k}$ and $\boldsymbol{X}_{m}(t)=\sum_{k=0}^{m} \Gamma_{k}(t) \mathbf{b}_{k}$ for IVP (1) are:

$\mathbf{a}_{0}=x_{0}$,

$\mathbf{a}_{k+1}=A \mathbf{a}_{k}+\varepsilon \boldsymbol{f}^{(k)}(0)$,

$\mathbf{b}_{0}=\boldsymbol{x}_{0}$,

$\mathbf{b}_{k+1}=\varepsilon \boldsymbol{f}^{(k)}(0)=\varepsilon \mathbf{c}_{k+1}$,

so:

$\boldsymbol{X}_{m}(t)=\Gamma_{0}(t) \boldsymbol{x}_{0}+\varepsilon \sum_{k=1}^{m} \Gamma_{k}(t) \boldsymbol{f}^{(k-1)}(0)$.

By inserting (34) into (1), we get the residual:

$\mathbf{R}_{m}(t)=\varepsilon \boldsymbol{f}(t)-\left(\boldsymbol{X}_{m}^{\prime}(t)-A \boldsymbol{X}_{m}(t)\right)$

$=\varepsilon \sum_{k=m}^{\infty} \frac{t^{k}}{k !} \boldsymbol{f}^{(k)}(0)$.

Furthermore, by taking into account that:

$\boldsymbol{x}_{m}^{\prime}(t)-A \boldsymbol{x}_{m}(t)$

$=\sum_{k=0}^{m-1}\left(\Gamma_{k}(t)-A \Gamma_{k+1}(t)\right)\left(\mathbf{a}_{k+1}-A \mathbf{a}_{k}\right)$

$-\left(\Gamma_{m}(t)-A \Gamma_{m+1}(t)\right) A \mathbf{a}_{m}$,

and by using (11), the residual corresponding to $\mathbf{x}_{m}(t)$ is given by:

$\mathbf{r}_{m}(t)=\varepsilon \boldsymbol{f}(t)-\left(\boldsymbol{x}_{m}^{\prime}(t)-A \boldsymbol{x}_{m}(t)\right)$

$=\varepsilon \sum_{k=m}^{\infty} \frac{t^{k}}{k !} \boldsymbol{f}^{(k)}(0)+\frac{t^{m}}{m !} A \mathbf{a}_{m}$.

Significant conclusions can be drawn from the expressions $\mathbf{r}_{m}(t)$ and $\mathbf{R}_{m}(t)$. In $\mathbf{R}_{m}(t)$, the perturbation parameter $\varepsilon$ is a factor of the residual, therefore $\mathbf{R}_{m}(t)$ is small with $\varepsilon$. On the contrary the residual $\mathbf{r}_{m}(t)$ splits into the sum of two quantities of which only one is proportional to $\varepsilon$; so in general $\mathbf{r}_{m}(t)$ is not small with $\varepsilon$. If $\varepsilon=0$, the Taylor series method produces a truncation error and yet the $\Gamma$-functions method, using just the first term, can accurately integrate the system of differential equations $[3,5]$.

\section{A NUMERICAL INTEGRATION METHOD BASED ON $\Gamma$-FUNCTIONS}

Following a similar procedure to the one described in [2, $5]$, in order to integrate IVP (1) using the $\Gamma$-functions method, the function $\boldsymbol{f}(\boldsymbol{x}, t)$ is expanded as follows:

$\boldsymbol{f}(\boldsymbol{x}, t)=\sum_{k=0}^{\infty} \frac{t^{k}}{k !} \mathbf{c}_{k+1}$,

and the solution, in terms of $\Gamma$-functions, is given by 
$\boldsymbol{x}(t)=\Gamma_{0}(t) \boldsymbol{x}_{0}+\varepsilon \sum_{k=1}^{\infty} \Gamma_{k}(t) \mathbf{c}_{k}$

where $\mathbf{c}_{k}$ are the derivatives of the perturbation function

$$
\boldsymbol{f}(\boldsymbol{x}(t), t)=\boldsymbol{g}(t)=\left(g_{1}(t), \ldots, g_{m}(t)\right),
$$

Denoting the point mesh in $[a, b]$ as $t_{i}=t_{i-1}+h_{i}, i=1,2, \ldots$, and the approximation to $\boldsymbol{x}\left(t_{i}\right)$ as $\boldsymbol{x}_{i}$, and assuming that we have calculated an approximation to the solution on point $t_{n}$, which we will call $\boldsymbol{x}_{n}$, it can be verified that

$\boldsymbol{x}^{\prime}=A \boldsymbol{x}+\boldsymbol{\varepsilon} \boldsymbol{f}(\boldsymbol{x}, t)$,

$\boldsymbol{x}\left(t_{n}\right)=\boldsymbol{x}_{n}$.

In order to obtain an approximation to the solution $\boldsymbol{x}\left(t_{n+1}\right)$, we switch the independent variable $\tau=t-t_{n}$, therefore (41) becomes:

$\boldsymbol{x}^{\prime}=A \boldsymbol{x}+\boldsymbol{\varepsilon} \boldsymbol{f}\left(\boldsymbol{x}, \tau+t_{n}\right)$,

$\boldsymbol{x}(0)=\boldsymbol{x}_{n}$,

which leads back to the initial situation.

The recurrence relation is used to calculate the expansion coefficients for:

$\boldsymbol{f}\left(\boldsymbol{x}(\tau), \tau+t_{n}\right)=\sum_{k=0}^{\infty} \frac{\tau^{k}}{k !} \mathbf{c}_{k+1}$,

with

$\boldsymbol{c}_{k+1}=\frac{d^{k} \boldsymbol{g}(0)}{d \tau^{k}}=\frac{d^{k} \boldsymbol{g}\left(t_{n}\right)}{d t^{k}}$,

and having calculated the necessary $\Gamma$-functions, it is possible to express the approximation to the solution at point $t_{\mathrm{n}+1}$ as follows:

$\boldsymbol{x}_{n+1}=\Gamma_{0}\left(h_{n+1}\right) \boldsymbol{x}_{n}+\varepsilon \sum_{j=1}^{p} \Gamma_{j}\left(h_{n+1}\right) \boldsymbol{g}^{(j-1)}\left(t_{n}\right)$,

which constitutes the numerical integration method for perturbed linear differential equations, on the basis of $\Gamma$ functions.

In certain cases, an alternative to switching the independent variable $\tau=t-t_{n}$ is the technique used by Steffensen [9], which involves some auxiliary variables which enable the system's perturbation function to be transformed into a function that does not depend explicitly on time or which transforms the system into a non-perturbed system, achieving integration in this case with just one $\Gamma$-function.

\section{RESOLUTION OF STIFF PROBLEMS}

In this section we present three examples showing the behaviour of the $\Gamma$-functions series method, against the known codes:
LSODE methods, causes a numerical solution to be found using the Livermore Stiff ODE solver. It solves stiff and nonstiff systems. It uses Adams methods (predictorcorrector) in the nonstiff case, and Backward Differentiation Formula (BDF) in the stiff case.

GEAR causes a numerical solution to be found by way of a Burlirsch-Stoer rational extrapolation method. The method has higher precision and calculation efficiency, especially in solving stiff differential equations.

MGEAR [msteppart] is a multi-step method suitable for stiff systems.

Using in the last ones the implementations of MAPLE V to ensure that the results are not distorted by a deficient programation that favours the new code.

\section{Problem I}

Let us consider the following stiff problem, which appears in [11-13]:

$\left\{\begin{array}{l}x_{1}^{\prime}(t)=-2 x_{1}(t)+x_{2}(t)+2 \sin (t) \\ x_{2}^{\prime}(t)=-(\beta+2) x_{1}(t)+(\beta+1)\left(x_{2}(t)-\cos (t)+\sin (t)\right),\end{array}\right.$

with $A=\left(\begin{array}{cc}-2 & 1 \\ -(\beta+2) & \beta+1\end{array}\right), \quad \varepsilon=1, \quad$ initial conditions $x_{1}(0)=2, x_{2}(0)=3$ and solution, independent of $\beta$ :

$x_{1}(t)=2 e^{-t}+\sin (t)$,

$x_{2}(t)=2 e^{-t}+\cos (t)$,

The eigenvalues of the system are -1 and $\beta$, which enables its degree of stiffness to be regulated. For the case $\beta=-1000$, the following stiff problem is obtained, proposed in [14]:

$\left(\begin{array}{l}x_{1}^{\prime}(t) \\ x_{2}^{\prime}(t)\end{array}\right)=\left(\begin{array}{cc}-2 & 1 \\ 998 & -999\end{array}\right)\left(\begin{array}{l}x_{1}(t) \\ x_{2}(t)\end{array}\right)+\left(\begin{array}{c}2 \sin (t) \\ 999(\cos (t)-\sin (t))\end{array}\right)$,

$x_{1}(0)=2, x_{2}(0)=3$.

Considering variables $x_{3}(t)=\sin (t), \quad x_{4}(t)=\cos (t)$, it is possible to tackle the following non-perturbed problem: $\left(\begin{array}{l}x_{1}^{\prime}(t) \\ x_{2}^{\prime}(t) \\ x_{3}^{\prime}(t) \\ x_{4}^{\prime}(t)\end{array}\right)=\left(\begin{array}{cccc}-2 & 1 & 2 & 0 \\ 998 & -999 & -999 & 999 \\ 0 & 0 & 0 & 1 \\ 0 & 0 & -1 & 0\end{array}\right)\left(\begin{array}{l}x_{1}(t) \\ x_{2}(t) \\ x_{3}(t) \\ x_{4}(t)\end{array}\right)$,
$x_{0}=\left(\begin{array}{lllll}x_{1}(0) & x_{2}(0) & x_{3}(0) & x_{4}(0)\end{array}\right)^{T}=\left(\begin{array}{llll}2 & 3 & 0 & 1\end{array}\right)^{T}$.

The introduction of new variables increases the dimension of the problem, but enables its integration using just the first of the $\Gamma$-functions, $\Gamma_{0}$.

Let $\boldsymbol{x}(t)$ be the solution to the above problem, which we assume to be analytical:

$$
\begin{aligned}
& \left(x_{1}(t) \quad x_{2}(t) \quad x_{3}(t) \quad x_{4}(t)\right)^{T}= \\
& \left(\sum_{k=0}^{\infty} \frac{t^{k}}{k !} a_{k, 1} \quad \sum_{k=0}^{\infty} \frac{t^{k}}{k !} a_{k, 2} \quad \sum_{k=0}^{\infty} \frac{t^{k}}{k !} a_{k, 3} \quad \sum_{k=0}^{\infty} \frac{t^{k}}{k !} a_{k, 4}\right)^{T},
\end{aligned}
$$


and when it is replaced in (49), we get:

$\sum_{k=0}^{\infty} \frac{t^{k}}{k !}\left(\begin{array}{l}a_{k+1,1} \\ a_{k+1,2} \\ a_{k+1,3} \\ a_{k+1,4}\end{array}\right)$
$=\sum_{k=0}^{\infty} \frac{t^{k}}{k !}\left(\begin{array}{cccc}-2 & 1 & 2 & 0 \\ 998 & -999 & -999 & 999 \\ 0 & 0 & 0 & 1 \\ 0 & 0 & -1 & 0\end{array}\right)\left(\begin{array}{c}a_{k, 1} \\ a_{k, 2} \\ a_{k, 3} \\ a_{k, 4}\end{array}\right)$.

Identifying the coefficients gives

$$
\left(\begin{array}{l}
a_{k+1,1} \\
a_{k+1,2} \\
a_{k+1,3} \\
a_{k+1,4}
\end{array}\right)=\left(\begin{array}{cccc}
-2 & 1 & 2 & 0 \\
998 & -999 & -999 & 999 \\
0 & 0 & 0 & 1 \\
0 & 0 & -1 & 0
\end{array}\right)\left(\begin{array}{c}
a_{k, 1} \\
a_{k, 2} \\
a_{k, 3} \\
a_{k, 4}
\end{array}\right) \text {, }
$$

with $k=0,1, \ldots$

By defining a new sequence of coefficients as:

$\mathbf{b}_{0}=\mathbf{a}_{0}=\boldsymbol{x}_{0}$,

$\mathbf{b}_{k}=\mathbf{a}_{k}-\left(\begin{array}{cccc}-2 & 1 & 2 & 0 \\ 998 & -999 & -999 & 999 \\ 0 & 0 & 0 & 1 \\ 0 & 0 & -1 & 0\end{array}\right) \mathbf{a}_{k-1}$,

with $k=1,2, \ldots$.

Hence, it is possible to express the solution to the problem in terms of $\Gamma$-functions:

$$
\boldsymbol{x}(t)=\sum_{k=0}^{\infty} \Gamma_{k}(t)\left(\begin{array}{l}
b_{k, 1} \\
b_{k, 2} \\
b_{k, 3} \\
b_{k, 4}
\end{array}\right),
$$

and having obtained the value of the $\Gamma$-functions; $\Gamma_{0}(t)=e^{A t}$ matrix has been calculate by the Richardsontype linear elimination procedure $[15,16]$; denoting by $\boldsymbol{x}_{1}$ the approximation to $\boldsymbol{x}(h)$, this approximate solution is given by:

$$
\boldsymbol{x}_{1}=\sum_{k=0}^{m} \Gamma_{k}(h)\left(\begin{array}{l}
b_{k, 1} \\
b_{k, 2} \\
b_{k, 3} \\
b_{k, 4}
\end{array}\right)=\Gamma_{0}(h)\left(\begin{array}{l}
b_{0,1} \\
b_{0,2} \\
b_{0,3} \\
b_{0,4}
\end{array}\right) \text {. }
$$

In order to calculate $\boldsymbol{x}_{2}$ (i.e. the approximation to $\boldsymbol{x}(2 h)$ ), the same process is carried out taking $\boldsymbol{x}_{1}$ as the initial value.

Each step is completed using the following algorithm: $\mathbf{a}_{0}=\boldsymbol{x}_{i}$,

$$
\begin{aligned}
& \left(\begin{array}{l}
a_{k+1,1} \\
a_{k+1,2} \\
a_{k+1,3} \\
a_{k+1,4}
\end{array}\right)=\left(\begin{array}{cccc}
-2 & 1 & 2 & 0 \\
998 & -999 & -999 & 999 \\
0 & 0 & 0 & 1 \\
0 & 0 & -1 & 0
\end{array}\right)\left(\begin{array}{c}
a_{k, 1} \\
a_{k, 2} \\
a_{k, 3} \\
a_{k, 4}
\end{array}\right) \text { for } k=0, \ldots, \\
& \mathbf{b}_{0}=\mathbf{a}_{0}, \\
& \mathbf{b}_{k}=\mathbf{a}_{k}-\left(\begin{array}{cccc}
-2 & 1 & 2 & 0 \\
998 & -999 & -999 & 999 \\
0 & 0 & 0 & 1 \\
0 & 0 & -1 & 0
\end{array}\right) \mathbf{a}_{k-1} \text { for } k=1, \ldots, \\
& \boldsymbol{x}_{i+1}=\sum_{k=0}^{m} \Gamma_{k}(h)\left(\begin{array}{l}
b_{k, 1} \\
b_{k, 2} \\
b_{k, 3} \\
b_{k, 4}
\end{array}\right)=\Gamma_{0}(h)\left(\begin{array}{l}
b_{0,1} \\
b_{0,2} \\
b_{0,3} \\
b_{0,4}
\end{array}\right) \text {. }
\end{aligned}
$$

Fig. (1) and Fig. (2) show the results obtained, with 40 and 60 digits, respectively, when the logarithm of the relative error vs $t$ of the $\Gamma$-functions method, using just one $\Gamma$ function, $\Gamma_{0}$, step size $h=0.1$ and 1000 iterations, is compared with the LSODE (tol $\left.=10^{-16}\right)$, MGEAR (errorper = Float $\left.\left(1,10^{-16}\right)\right)$ and GEAR $\left(\right.$ errorper $\left.=\operatorname{Float}\left(1,10^{-16}\right)\right)$.

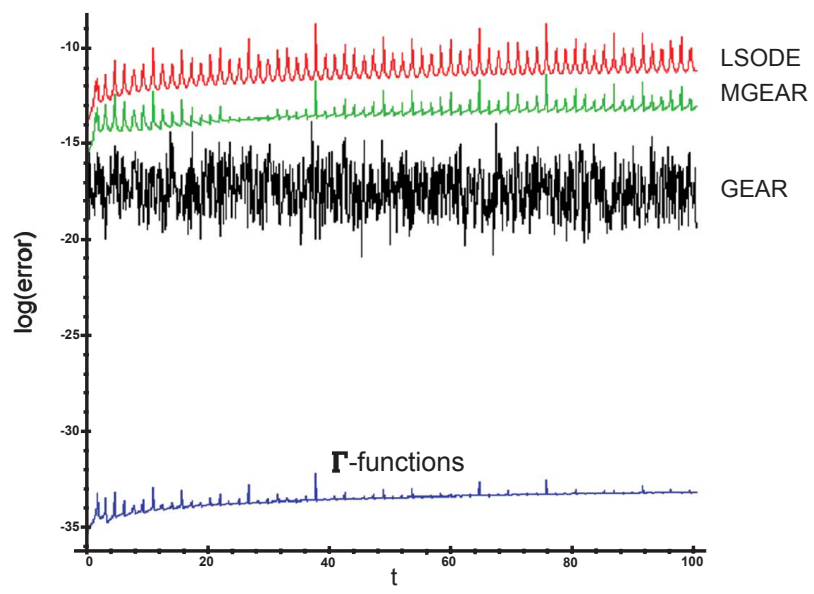

Fig. (1). Lambert's problem, $h=0.1,40$ digits, one $\Gamma$-function.

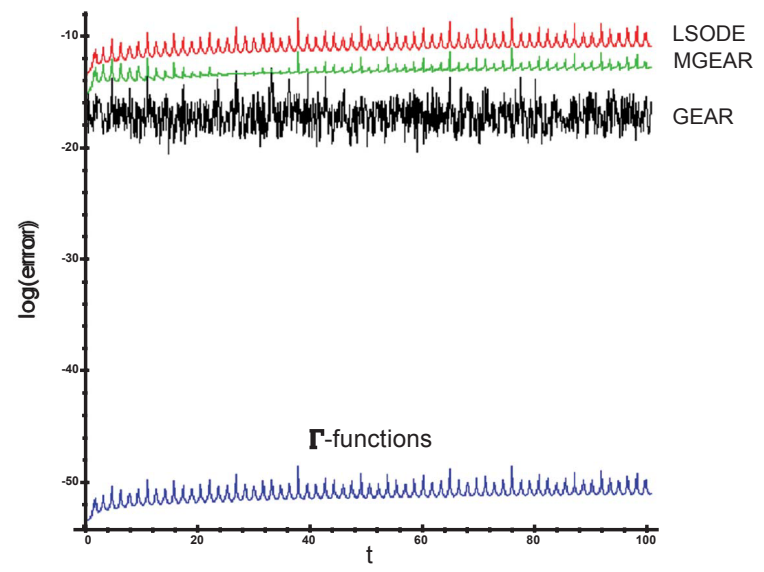

Fig. (2). Lambert's problem, $h=0.1,60$ digits, one $\Gamma$-function. 
The error is taken to be the difference between the solution of the several methods and the exact solution. Is noted the well behaviour of the $\Gamma$-function series method, maintaining the error bounded throughout the integration.

Fig. (1) and Fig. (2) show that with the same conditions, increasing the number of digits, the error decreases.

\section{Problem II}

Let us consider the following stiff test problem, proposed in general terms in [17]:

$\left\{\begin{array}{l}x_{1}^{\prime}(t)=-\left(2+\varepsilon^{-1}\right) x_{1}(t)+\varepsilon^{-1} x_{2}^{2}(t) \\ x_{2}^{\prime}(t)=x_{1}(t)-x_{2}(t)\left(1+x_{2}(t)\right)\end{array}\right.$,

with the initial conditions $x_{1}(0)=1, x_{2}(0)=1$ and solution, independent of $\varepsilon$ :

$x_{1}(t)=e^{-2 t}, x_{2}(t)=e^{-t}$

the eigenvalues of the system are $-1,-\left(2+\varepsilon^{-1}\right)$, which enables the degree of stiffness to be regulated. A particularisation for $\varepsilon=10^{-1}$ is represented in $[18,19]$ sets forth the Kaps problem for $\varepsilon=10^{-3}$ obtaining the following stiff system:

$$
\begin{aligned}
& \left(\begin{array}{l}
x_{1}^{\prime}(t) \\
x_{2}^{\prime}(t)
\end{array}\right)=\left(\begin{array}{cc}
-1002 & 0 \\
1 & -1
\end{array}\right)\left(\begin{array}{l}
x_{1}(t) \\
x_{2}(t)
\end{array}\right)+\left(\begin{array}{c}
1000 x_{2}^{2}(t) \\
-x_{2}^{2}(t)
\end{array}\right), \\
& \boldsymbol{x}_{0}=\left(\begin{array}{ll}
x_{1}(0) & \left.x_{2}(0)\right)^{T}=\left(\begin{array}{ll}
1 & 1
\end{array}\right)^{T} .
\end{array}\right.
\end{aligned}
$$

Let $\boldsymbol{x}(t)$ be the solution to the abovementioned problem, which we assume to be analytical:

$\left(x_{1}(t) \quad x_{2}(t)\right)^{T}$

$=\left(\sum_{k=0}^{\infty} \frac{t^{k}}{k !} a_{k, 1} \quad \sum_{k=0}^{\infty} \frac{t^{k}}{k !} a_{k, 2}\right)^{T}$.

By substituting (60) in (59) and applying the Cauchy's rule for the series product, we get:

$$
\begin{aligned}
& \sum_{k=0}^{\infty} \frac{t^{k}}{k !}\left(\begin{array}{l}
a_{k+1,1} \\
a_{k+1,2}
\end{array}\right)=\sum_{k=0}^{\infty} \frac{t^{k}}{k !}\left(\begin{array}{cc}
-1002 & 0 \\
1 & -1
\end{array}\right)\left(\begin{array}{l}
a_{k, 1} \\
a_{k, 2}
\end{array}\right) \\
& +\sum_{k=0}^{\infty} \frac{t^{k}}{k !}\left(\begin{array}{c}
1000 \sum_{i=0}^{k}\left(\begin{array}{l}
k \\
i
\end{array}\right) a_{i, 2} a_{k-i, 2} \\
-\sum_{i=0}^{k}\left(\begin{array}{c}
k \\
i
\end{array}\right) a_{i, 2} a_{k-i, 2}
\end{array}\right) .
\end{aligned}
$$

Identifying coefficients gives

$$
\begin{aligned}
& \left(\begin{array}{l}
a_{k+1,1} \\
a_{k+1,2}
\end{array}\right)=\left(\begin{array}{cc}
-1002 & 0 \\
1 & -1
\end{array}\right)\left(\begin{array}{l}
a_{k, 1} \\
a_{k, 2}
\end{array}\right) \\
& +\left(\begin{array}{c}
1000 \sum_{i=0}^{k}\left(\begin{array}{l}
k \\
i
\end{array}\right) a_{i, 2} a_{k-i, 2} \\
-\sum_{i=0}^{k}\left(\begin{array}{l}
k \\
i
\end{array}\right) a_{i, 2} a_{k-i, 2}
\end{array}\right),
\end{aligned}
$$

an expression that enables us to define the following succession of coefficients:

$$
\begin{aligned}
& \mathbf{b}_{0}=\mathbf{a}_{0}=\boldsymbol{x}_{0}, \\
& \mathbf{b}_{k}=\mathbf{a}_{k}-\left(\begin{array}{cc}
-1002 & 0 \\
1 & -1
\end{array}\right) \mathbf{a}_{k-1} \\
& =\left(\begin{array}{c}
1000 \sum_{i=0}^{k-1}\left(\begin{array}{c}
k-1 \\
i
\end{array}\right) a_{i, 2} a_{k-i-1,2} \\
-\sum_{i=0}^{k-1}\left(\begin{array}{c}
k-1 \\
i
\end{array}\right) a_{i, 2} a_{k-i-1,2}
\end{array}\right) \text { with } k=1,2, \ldots .
\end{aligned}
$$

Hence, it is now possible to express the solution to (59) in terms of $\Gamma$-functions:

$$
\boldsymbol{x}(t)=\sum_{k=0}^{\infty} \Gamma_{k}(t)\left(\begin{array}{l}
b_{k, 1} \\
b_{k, 2}
\end{array}\right) .
$$

$\Gamma_{0}(t)=e^{A t}$ matrix has been calculate by the Richardsontype linear elimination procedure $[15,16]$ and $\Gamma_{k}(t)$ for $k=1, \ldots$, are calculated by recurrence relation (11).

Denoting the approximation to $\boldsymbol{x}(h)$, as $\boldsymbol{x}_{1}$, said approximation to the solution is given as follows:

$$
\boldsymbol{x}_{1}=\sum_{k=0}^{m} \Gamma_{k}(h)\left(\begin{array}{l}
b_{k, 1} \\
b_{k, 2}
\end{array}\right) \text {. }
$$

In order to calculate $\boldsymbol{x}_{2}$, the same process is carried out, taking $\boldsymbol{x}_{1}$ as the initial value.

Each step is completed using the following algorithm:

$\mathbf{a}_{0}=\boldsymbol{x}_{i}$

$\left(\begin{array}{c}a_{k+1,1} \\ a_{k+1,2}\end{array}\right)=\left(\begin{array}{cc}-1002 & 0 \\ 1 & -1\end{array}\right)\left(\begin{array}{l}a_{k, 1} \\ a_{k, 2}\end{array}\right)+\left(\begin{array}{c}1000 \sum_{i=0}^{k}\left(\begin{array}{l}k \\ i\end{array}\right) a_{i, 2} a_{k-i, 2} \\ -\sum_{i=0}^{k}\left(\begin{array}{l}k \\ i\end{array}\right) a_{i, 2} a_{k-i, 2}\end{array}\right)$ for $k=0, \ldots$

$\mathbf{b}_{0}=\mathbf{a}_{0}$,

$\mathbf{b}_{k}=\mathbf{a}_{k}-\left(\begin{array}{cc}-1002 & 0 \\ 1 & -1\end{array}\right) \mathbf{a}_{k-1}$

$=\left(\begin{array}{c}1000 \sum_{i=0}^{k-1}\left(\begin{array}{c}k-1 \\ i\end{array}\right) a_{i, 2} a_{k-i-1,2} \\ -\sum_{i=0}^{k-1}\left(\begin{array}{c}k-1 \\ i\end{array}\right) a_{i, 2} a_{k-i-1,2}\end{array}\right)$,

for $k=1, \ldots$.

$$
\boldsymbol{x}_{i+1}=\sum_{k=0}^{m} \Gamma_{k}(h)\left(\begin{array}{l}
b_{k, 1} \\
b_{k, 2}
\end{array}\right) .
$$

Fig. (3) shows the result obtained when the logarithm of the relative error vs $t$ of the $16 \Gamma$-functions method, step size 
$h=0.01$ and 1000 iterations, is compared with the LSODE $\left(\right.$ tol $\left.=10^{-20}\right)$ and GEAR $\left(\right.$ errorper $=$ Float $\left.\left(1,10^{-20}\right)\right)$ methods, with 40 digits.

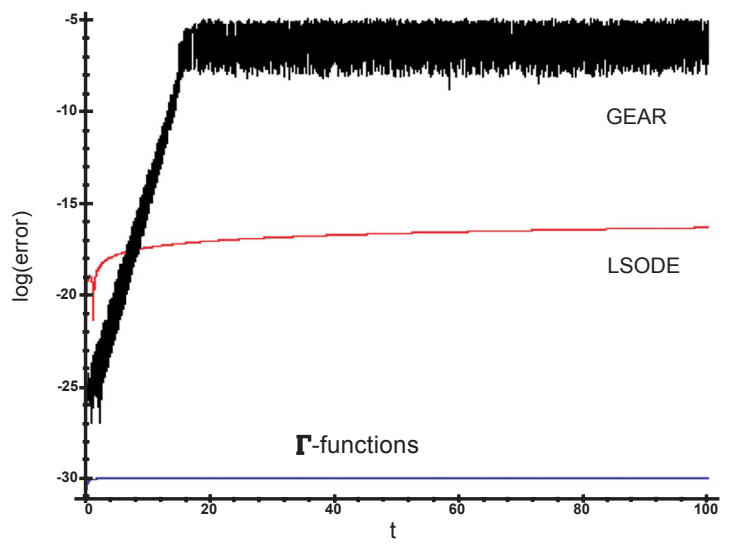

Fig. (3). Kaps' problem, $h=0.01,40$ digits, sixteen $\Gamma$-functions.

The error is taken to be the difference between the solution of the several methods and the exact solution. Is noted the well behaviour of the $\Gamma$-function series method, maintaining the error bounded throughout the integration.

\section{Problem III}

This example shows an application of the $\Gamma$-functions method to a problem of quasiperiodic orbits studied by [20], which can also be found in [13, 21-23], among others.

Let

$x^{\prime \prime}(t)+x(t)=10^{-3} e^{i t}$,

with the initial conditions:

$x(0)=1, x^{\prime}(0)=0.9995 i$,

for which the analytical solution is:

$x(t)=\left(1-5 \cdot 10^{-4} i t\right) e^{i t}$

$=\left(\cos (t)+5 \cdot 10^{-4} t \sin (t)\right)$

$+i\left(\sin (t)-5 \cdot 10^{-4} t \cos (t)\right)$

The solution represents motion on a perturbation of a circular orbit in the complex plane. The problem may be solved either as a single equation in complex arithmetic or a pair of uncoupled equations.

Noting

$x(t)=u(t)+i v(t)$

and by substituting in (67), we get the following second order system:

$\left\{\begin{array}{l}u^{\prime \prime}(t)+u(t)=10^{-3} \cos (t), \\ v^{\prime \prime}(t)+" v(t)=10^{-3} \sin (t),\end{array}\right.$ with the initial conditions:

$u(0)=1, u^{\prime}(0)=0, v(0)=0, v^{\prime}(0)=0.9995$.

By defining the variables:

$x_{1}(t)=u(t), x_{2}(t)=u^{\prime}(t)$,
$x_{3}(t)=v(t), x_{4}(t)=v^{\prime}(t)$.

(70) becomes the system of first order linear equations:

$$
\left\{\begin{array}{l}
x_{1}^{\prime}(t)=x_{2}(t), \\
x_{2}^{\prime}(t)=-x_{1}(t)+10^{-3} \cos (t), \\
x_{3}^{\prime}(t)=x_{4}(t), \\
x_{4}^{\prime}(t)=-x_{3}(t)+10^{-3} \sin (t),
\end{array}\right.
$$

with the initial conditions:

$x_{1}(0)=1, x_{2}(0)=0, x_{3}(t)=0, x_{4}(0)=0.9995$.

The auxiliary variables $[9,10]$ :

$x_{5}(t)=\cos (t), x_{6}(t)=\sin (t)$,

enable (73) to be expressed as a non-perturbed first order linear system:

$$
\left(\begin{array}{l}
x_{1}^{\prime}(t) \\
x_{2}^{\prime}(t) \\
x_{3}^{\prime}(t) \\
x_{4}^{\prime}(t) \\
x_{5}^{\prime}(t) \\
x_{6}^{\prime}(t)
\end{array}\right)=\left(\begin{array}{cccccc}
0 & 1 & 0 & 0 & 0 & 0 \\
-1 & 0 & 0 & 0 & 10^{-3} & 0 \\
0 & 0 & 0 & 1 & 0 & 0 \\
0 & 0 & -1 & 0 & 0 & 10^{-3} \\
0 & 0 & 0 & 0 & 0 & -1 \\
0 & 0 & 0 & 0 & 1 & 0
\end{array}\right)\left(\begin{array}{c}
x_{1}(t) \\
x_{2}(t) \\
x_{3}(t) \\
x_{4}(t) \\
x_{5}(t) \\
x_{6}(t)
\end{array}\right),
$$

with the

initial

conditions:

$\boldsymbol{x}_{0}=\left(x_{1}(0) \quad x_{2}(0) \quad x_{3}(0) \quad x_{4}(0) \quad x_{5}(0) \quad x_{6}(0)\right)^{T}$

$=\left(\begin{array}{llllll}1 & 0 & 0 & 0.9995 & 1 & 0\end{array}\right)^{T}$.

Although this procedure increases the dimension of the problem, it enables integration using just the first of the $\Gamma$ functions, $\Gamma_{0}$.

Let $\boldsymbol{x}(t)$ be the solution to the previous problem, which we assume to be analytical:

$x_{i}(t)=\sum_{k=0}^{\infty} \frac{t^{k}}{k !} a_{k, i} \quad i=1, \ldots, 6$.

By substituting (78) in (76) and identifying the coefficients, we get:

$\left(\begin{array}{l}a_{k+1,1} \\ a_{k+1,2} \\ a_{k+1,3} \\ a_{k+1,4} \\ a_{k+1,5} \\ a_{k+1,6}\end{array}\right)=\left(\begin{array}{cccccc}0 & 1 & 0 & 0 & 0 & 0 \\ -1 & 0 & 0 & 0 & 10^{-3} & 0 \\ 0 & 0 & 0 & 1 & 0 & 0 \\ 0 & 0 & -1 & 0 & 0 & 10^{-3} \\ 0 & 0 & 0 & 0 & 0 & -1 \\ 0 & 0 & 0 & 0 & 1 & 0\end{array}\right)\left(\begin{array}{l}a_{k, 1} \\ a_{k, 2} \\ a_{k, 3} \\ a_{k, 4} \\ a_{k, 5} \\ a_{k, 6}\end{array}\right)$,

an expression that enables us to define the following sequence: 
$\mathbf{b}_{0}=\mathbf{a}_{0}=\boldsymbol{x}_{0}$,

$\mathbf{b}_{k}=\mathbf{a}_{k}-\left(\begin{array}{cccccc}0 & 1 & 0 & 0 & 0 & 0 \\ -1 & 0 & 0 & 0 & 10^{-3} & 0 \\ 0 & 0 & 0 & 1 & 0 & 0 \\ 0 & 0 & -1 & 0 & 0 & 10^{-3} \\ 0 & 0 & 0 & 0 & 0 & -1 \\ 0 & 0 & 0 & 0 & 1 & 0\end{array}\right) \mathbf{a}_{k-1}$,

with $k=1,2, \ldots$.

Having obtained the value of the $\Gamma$-functions; $\Gamma_{0}(t)=e^{A t}$ matrix has been calculate by the Richardsontype linear elimination procedure $[15,16]$; denoting the approximation to $\boldsymbol{x}(h)$, as $\boldsymbol{x}_{1}$, this approximation to the solution would be given as follows:

$\boldsymbol{x}_{1}=\sum_{k=0}^{m} \Gamma_{k}(h)\left(b_{k, 1}, b_{k, 2}, b_{k, 3}, b_{k, 4}, b_{k, 5}, b_{k, 6}\right)^{T}$.

In order to calculate $\boldsymbol{x}_{2}$, the same process is carried out, taking $\boldsymbol{x}_{1}$ as the initial value.

Each step is completed using the following algorithm:

$\mathbf{a}_{0}=\boldsymbol{x}_{i}$,

$\mathbf{a}_{k+1}=\left(\begin{array}{cccccc}0 & 1 & 0 & 0 & 0 & 0 \\ -1 & 0 & 0 & 0 & 10^{-3} & 0 \\ 0 & 0 & 0 & 1 & 0 & 0 \\ 0 & 0 & -1 & 0 & 0 & 10^{-3} \\ 0 & 0 & 0 & 0 & 0 & -1 \\ 0 & 0 & 0 & 0 & 1 & 0\end{array}\right) \mathbf{a}_{k-1}$

for $k=0, \ldots$.

$\mathbf{b}_{0}=\mathbf{a}_{0}$,

$\mathbf{b}_{k}=\mathbf{a}_{k}-\left(\begin{array}{cccccc}0 & 1 & 0 & 0 & 0 & 0 \\ -1 & 0 & 0 & 0 & 10^{-3} & 0 \\ 0 & 0 & 0 & 1 & 0 & 0 \\ 0 & 0 & -1 & 0 & 0 & 10^{-3} \\ 0 & 0 & 0 & 0 & 0 & -1 \\ 0 & 0 & 0 & 0 & 1 & 0\end{array}\right) \quad$ for $k=1, \ldots$.

$\boldsymbol{x}_{i+1}=\sum_{k=0}^{m} \Gamma_{k}(h)\left(b_{k, 1}, b_{k, 2}, b_{k, 3}, b_{k, 4}, b_{k, 5}, b_{k, 6}\right)^{T}$,

Fig. (4) shows the result obtained when the logarithm of the relative error vs $t$ of the $\Gamma$-functions model, with just one $\Gamma$-function, $\Gamma_{0}$, step size $h=0.1$ and 1000 iterations, is compared with the LSODE (tol $=10^{-16}$ ), MGEAR (errorper $\left.=\operatorname{Float}\left(1,10^{-16}\right)\right)$ and GEAR (errorper $\left.=\operatorname{Float}\left(1,10^{-16}\right)\right)$ methods implemented in Maple V, with 60 digits.

Fig. (5) shows the same comparison as the previous figure but with a step size nine times greater, in other words $h=0.9$ and 100 iterations.

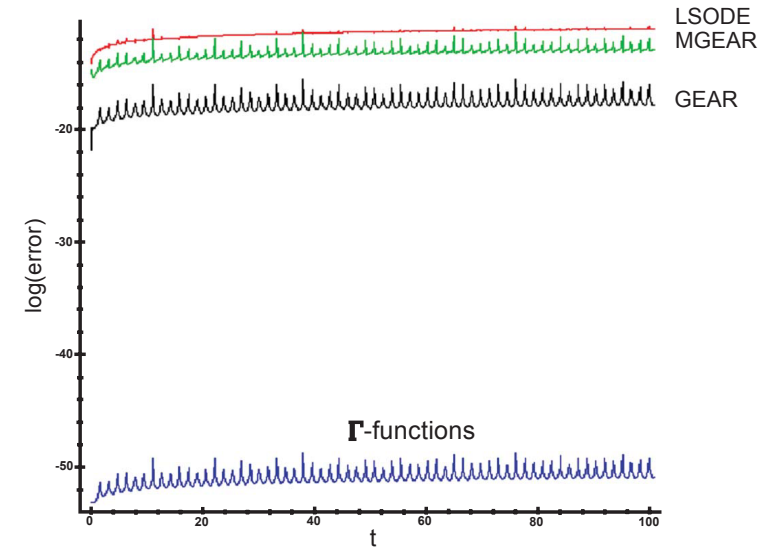

Fig. (4). Stiefel and Bettis problem, $h=0.1,60$ digits, one $\Gamma$-function.

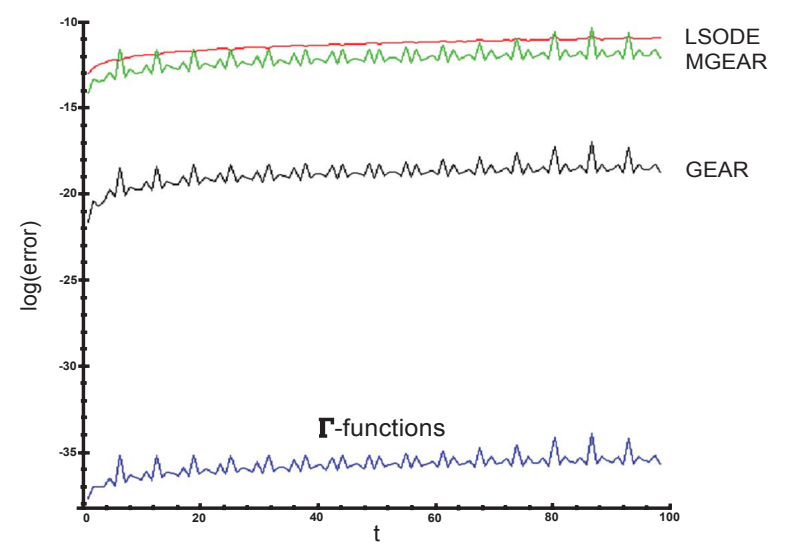

Fig. (5). Stiefel and Bettis problem, $h=0.9,60$ digits, one $\Gamma$ - function.

The error is taken to be the difference between the solution of the several methods and the exact solution. Is noted the well behaviour of the $\Gamma$-function series method, maintaining the error bounded throughout the integration.

Fig. (5) shows the well behaviour of the $\Gamma$-function series method, if it increase the step size.

\section{CONCLUSIONS}

The method here considered is the direct generalization of the algorithm for the integration of perturbed oscillators described in [2], to perturbed linear systems of differential equations; the good properties of this algorithm for equations are retained.

Numerical experiments confirm the strong performance of the numerical integration method, based on the Scheifele $\Gamma$-functions series; when treating stiff problems, it enables the non-perturbed problem to be accurately integrated using just the first term in the series. 
Furthermore, the method can be taken as the foundation for multistep numerical methods, also based on $\Gamma$-functions or some refinement such as $\mathrm{SMF}[4], \mathrm{SVF}[7,21]$ and $\mathrm{EI}_{P} \mathrm{PC}$ [8].

\section{REFERENCES}

[1] Stiefel EL, Scheifele G. Linear and regular celestial mechanics, Springer, New York 1971.

[2] García-Alonso F, Reyes JA, Villacampa Y. Integration of highly oscillatory problems through $G$-functions. Open Appl Math J 2007; 1: 9-14.

[3] Scheifele G. On numerical integration of perturbed linea oscillating systems. ZAMP 1971; 22: 186-210.

[4] Martín P, Ferrándiz JM. Behaviour of the SMF method for thenumerical integration of satellite orbits. Celestial Mech Dyn Astron $1995 ; 63: 29-40$.

[5] Martín P, Ferrándiz JM. Multistep numerical methods based on Scheifele G-functions with application to satellite dynamics. SIAM J Numer Anal 1997; $34:$ 359-75.

[6] Martín P, Ferrándiz JM. Numerical integration of perturbed linear systems. Appl Math 1999; 31: 183-9.

[7] Vigo-Aguiar J, Ferrándiz JM. Higher-order variable-step algorithms adapted to the accurate numerical integration of perturbed oscillators. Comput Phys 1998; 12 (5) : 467-70.

[8] Reyes JA, García-Alonso F, Ferrándiz JM, Vigo-Aguiar J. Numeric multistep variable methods for perturbed linear system integration. Appl Math Comput 2007; $190: 63-79$.

[9] Steffensen JF. On the differential equations of Hill in the theory of the motion of the moon. Acta Math 1955; $93: 169-77$.

[10] Steffensen JF. On the differential equations of Hill in the theory of the motion of the moon (II). Acta Math 1955; 95: 25-37.
[11] Ixaru L Gr, Vanden Berghe G, De Meyer H. Frequency evaluation in exponential fitting multistep algorithms for ODEs. J Comput Appl Math 2002; $140: 423-34$.

[12] Ixaru L Gr, Vanden Berghe G, De Meyer H. Exponentially fitted variable two-step $B D F$ algorithms for first order ODEs Comput Phys Commun 2003;100: 56-70.

[13] Van de Vyver H. Two-step hybrid methods adapted to the numerical integration of perturbed oscillators, arXiv:math/0612637v1 [math.NA] 21 Dec 2006

[14] Lambert JD. Numerical methods for ordinary differential systems. John Willey and Sons Ltd, New York 1991.

[15] Walz G. Approximation von Funktionen durch asymptotische Entwicklungen und Eliminationsprozeduren. Dissertation. Universität Mannheim 1987.

[16] Walz G. Computing the matrix exponential and other matrix functions. J Comput Appl Math 1988; 21: 119-23.

[17] Kaps P, Rosenbrock-type methods. In: Dahlquist G, Jeltsch R, Eds. Numerical methods for stiff initial value problems, Berich Nr. 9, Inst. für Geometrie und Praktische Mathematik der RWTH Aachen 1981.

[18] van der Houwen PJ, Sommeijer BP. The use of approximate factorization in stiff ODE solvers. Report MAS- R9732 CWI, Amsterdam 1997.

[19] Frank JE, Houwen PJ. Parallel iteration of the extended backward differentiation formulas. IMA J Numer Anal 2001; 21: 367-85.

[20] Stiefel E, Bettis DG. Stabilization of Cowell's method. Numer Math 1969; 13: 154-75.

[21] Vigo-Aguiar J, Ferrándiz JM. A general procedure for the adaptation of multistep algorithms to the integration of oscillatory problems. SIAM J Numer Anal 1998; 35(4): 1684-708.

[22] Simos TE, Vigo-Aguiar J. Exponentially fitted sympletic integrator. Phys Rev E 2003; 67: 1-7.

[23] Ramos JI. Piecewise-linearized methods for initial-value problems with oscillating solutions. Appl Math Comput 2006; 181: 123-46.

(C) Reyes et al.; Licensee Bentham Open.

This is an open access article distributed under the terms of the Creative Commons Attribution License (http://creativecommons.org/licenses/by/2.5/), which permits unrestrictive use, distribution, and reproduction in any medium, provided the original work is properly cited. 